\title{
Evaluation of the Sensitivity of Rapid Diagnostic Test (RDT) of Malaria in Different Age and Sex Groups Using Microscopy as a Gold Standard
}

\author{
Muktari Isa Shago ${ }^{1}$, Markus Kirwe Jiyil ${ }^{2}$, , Nazifi Alhaji Sani ${ }^{1}$, Garba Suleiman ${ }^{1}$ \\ ${ }^{1}$ Department of Chemistry, Yobe State University Damaturu, Damaturu, Nigeria \\ ${ }^{2}$ Department of Biochemistry, University of Jos, Jos, Nigeria \\ Email address: \\ eazziey.eazziey@gmail.com (M. I. Shago),kirwej@unijos.edu.ng (M. K. Jiyil) nazifialhajisani@gmail.com (N. A. Sani), \\ sulei2006@gmail.com (G. Suleiman) \\ ${ }^{*}$ Corresponding author
}

\section{To cite this article:}

Muktari Isa Shago, Markus Kirwe Jiyil, Nazifi Alhaji Sani, Garba Suleiman. Evaluation of the Sensitivity of Rapid Diagnostic Test (RDT) of Malaria in Different Age and Sex Groups Using Microscopy as a Gold Standard. International Journal of Infectious Diseases and Therapy. Vol. 5, No. 3, 2020, pp. 99-105. doi: 10.11648/j.ijidt.20200503.21

Received: February 18, 2020; Accepted: February 28, 2020; Published: September 16, 2020

\begin{abstract}
Malaria remains a public health problem in over 90 countries worldwide, especially in Africa where it is the cause of morbidity and mortality. The aim of the study is to compare the sensitivity of two rapid diagnostic test kits in the diagnosis of malaria among patients attending Yobe State Specialist Hospital Damaturu. Patients were screened for malaria using blood samples collected in an EDTA container. Patients were tested with one Histidine rich protein-2(HRP-2) RDT (SD-Bioline) and a combination of Histidine rich protein-2(HRP-2) and Parasite lactose dehydrogenase enzyme (pLDH) RDT (CareStart). Microscopy was used as a gold standard. Out of 200 participants enrolled and screened for malaria, $200(100 \%)$ tested positive by Microscopy, 159 (79.5\%), tested positive by SD-Bioline (HRP 2 RDT) while 139 (69.5\%), tested positive with the CareStart (pLDH RDT). Upon detection of the sensitivity by serially diluting the positive samples in other to decrease the parasite density; SD-Bioline was reactive at 1:4 dilution while CareStart was weekly reactive at this dilution. Although SD-Bioline showed to have a higher sensitivity than CareStart, both could be a suitable alternative to microscopy to screen endemic malaria in Nigeria.
\end{abstract}

Keywords: Diagnostic, Infection, Microscopy, Malaria, Yobe

\section{Introduction}

Malaria remains a public health problem in over 90 countries worldwide, especially in Africa where it is the cause of mortality mostly in children under 5 years. It accounts for $40 \%$ of Public Health expenditure, 30-50\% inpatients admissions and up to $50 \%$ of out-patients visits [1].

Malaria is a disease caused by various species of plasmodium ( $P$. falciparum, $P$. ovale, $P$. vivax, $P$. malariae and $P$. knowlesi) transmitted through the bite of female anopheles mosquito. The most serious and sometimes fatal type of malaria is caused by plasmodium falciparum (WHO, 2010). Malaria is a serious and sometimes life threatening tropical disease that spreads through parasites. Patients with severe falciparum malaria may develop liver and kidney failure, convulsions and coma. Although occasionally sever infection with $P$. vivax and $P$. ovale generally cause less severe illness, but the parasite can remain dormant in the liver for many months, causing the reoccurrence of the symptoms for many months or even years later [2].

Detection of sub-microscopic infections is crucial not only to effect prompt treatment of asymptomatic cases, but also to identify and clear potential reservoirs of transmission and to reduce malaria related morbidity and mortality [3]. Presumptive treatment of malaria based on clinical diagnosis is relatively cheap but it is unreliable due to overlapping symptoms with non-malarial infections caused by viruses or bacteria and could lead to multiple diagnosis as well [4].

Wrong diagnoses leads to wrong medication and hence many patients may leave the health facility without the right treatment. Rational prescription of anti-malarial drug is not only important in saving on the cost of expensive drugs but it also prevents drug 
overuse that might result in the development of resistance [5].

Malaria presents a diagnostic challenge to Medical Laboratory Personals in most malaria endemic countries [6]. The World Health Organization has opened a dialogue with Scientists, Clinicians and manufacturers on the realistic possibilities for developing accurate, sensitive, and cost effective rapid diagnostic test for malaria [7]. New technology has to be compared with accepted world health organization (WHO) standards that make comparisons of sensitivities between different methods. Majority of malaria cases are found in countries where cost effectiveness is an important factor and case of performance and training is a major consideration [8].

However the constitutive symptoms of malaria include fever, headache, weakness, myalgia, chills, dizziness, abdominal pain, diarrhea, nausea, vomiting, anorexia and pruritus [9].

Accuracy of a clinical diagnosis varies with the level of endemicity, season and age group. Malaria transmission, morbidity and mortality can be reduced if prompt diagnosis and adequate treatment is available [10]. Rapid diagnostic tests (RDTs) offer the potential to provide accurate and timely diagnosis to everyone at risk, reaching those previously unable to access good quality and qualitative microscopic services [11]. In malaria-endemic regions, the use of RDTs is very helpful for the effective use of antimalarial drugs as treatment is based on parasite diagnosis and not just fever alone in these region, a considerable proportion of these drugs have been wasted on patients with no malarial disease due to lack of prompt and accurate diagnosis [12].

Since 2010, WHO recommends that all suspected cases of malaria should be confirmed parasitological by microscopy or RDTs before treatments, irrespective of endemicity, season and age group [13]. Current interest in rapid diagnosis of malaria is focused primarily on detection of hisdtidine-rich protein-2 from Plasmodium falciparum and parasite specific lactate dehydrogenase for all species of plasmodium [14]. Its advantages lie in its ability to give rapid and similar result to non-specialized microscopist as well as the reduced requirement for specialized equipment and high man power training for its performance. It is the only current option in some areas for blood based diagnosis [15].

Microscopy was reported to detect about $75 \%$ of malaria infections in endemic areas, whereas in non-endemic areas this method has been reported to miss up to $88 \%$ of infections [16].

Furthermore, the level of expertise of technicians, quality of the equipment, and workload may lead to inaccurate estimates of parasite density and species differentiation [17].

\section{Materials and Methods}

\subsection{Study Area}

The study was carried out in Yobe State Specialist Hospital, which is located KM 3 along Gujba road, Damaturu. It is a state located in the North-Eastern Nigeria. It is the capital of Yobe State. The state was created on August 27, 1991. It is one of the 36 states in Nigeria which has 17 local government areas and a total land area of $3,366 \mathrm{~km}^{2}$ with total population of 88,014 as the last census data [18].

\subsection{Sample Size}

A convenient sample size of 200 was used.

\subsubsection{Study Population}

The study population include all patients with malaria attending Yobe State Specialist Hospital, Damaturu.

\subsubsection{Inclusion Criteria}

The criteria for inclusion are as follows;

i. Symptomatic patients.

ii. Patients within age group 1-60 years.

iii. Patients tested positive microscopically

\subsection{Exclusion Criteria}

Asymptomatic patients.

\subsection{Sample Collection, Processing and Preservation}

Blood samples were collected from patients suspected to have malaria by venipuncture into vacuum containers containing EDTA. After mixing, the blood samples were used to determine the plasmodia from the preparation of thick film for microscopy and for the RDTs assay.

\subsection{Preparation of Blood Films for Microscopy}

\subsubsection{Making of Thick Blood Film}

A small drop of blood was placed at the center of a grease free glass slide and spread with the edge of another slide in a repeated coil shape to a diameter approximately $2 \mathrm{~cm}$. The slide was labeled and left horizontally allowing it to air dry, protecting them from dust and flies [19].

\subsubsection{Staining of Thick Blood Film by Field's Stain}

An appropriate dilution of the stock Giemsa stain (about $10 \%$ ) was prepared using tap water ( $\mathrm{pH}$ of 7.1$)$. The stain was poured on the slide and allowed for 45 minutes. The stain was washed slowly under tap to remove excess stain. Undersurface of the slide was wiped with cotton wool and allowed to air-dry in a vertical position [20].

\subsubsection{Examination of Stained Blood Films for Parasite}

The search for malaria parasite on blood film was carried out under $\times 100$ oil immersion objective lens of a microscope after using one to two drops of immersion oil [21].

Positive result:

The presence of single malaria parasite qualified the sample as positive and progressed to parasite count.

Negative result:

No parasite found after examination of hundred high power fields [22].

\subsubsection{Test Procedure for RDT (Following Manufacturer's Instructions)}

Test device, buffer and specimen were allowed to equilibrate at room temperature $\left(10^{\circ} \mathrm{C}-30^{\circ} \mathrm{C}\right)$ prior to testing. The test cassette was removed from the foil pouch by 
tearing at the notch and then placed on level surface.

1. $5 \mu$ of whole blood was slowly added into the sample well (A)

2. Then 3 drops of clearing buffer was added to the buffer well (B)

3. As the test begins to work, a purple color moving across the result window in the center of the test device.

4. A result was read after 25 minutes. (Hopkins et al., 2008).

\subsubsection{Interpretation of Result}

Positive (+) Result:

Rose pink bands are visible in both the control region and the test region.

Negative (-) Result

A rose pink is visible in the control region and no color bands appear in the test region.

Invalid Result:
No visible band at all or there may be a visible band only in the test region but not in the control region.

\subsubsection{Detection and Estimation of Parasitemia Values}

Thick film was prepared from each of samples and stained using Giemsa stain. The films were examined microscopically for parasites, using parasite count method. Samples that show presence of parasites were quantified accordingly. The parasite numbers $/ \mu \mathrm{L}$ of blood was estimated by counting parasites against white blood cells. A part of the thick film where the white cells were evenly distributed and the parasites were well stained. Using the oil immersion objective, systematically 200 white blood cells (WBC) were counted and at the same time the numbers of parasites (asexual) were counted in each field covered. The number of parasites per $\mu \mathrm{L}$ of blood were estimated as follows:

$$
\text { Number of parasites } / \mathrm{Ml}=\frac{\text { Parasites counted against } 200 \mathrm{WBC} \times \text { Patient's WBC Count }}{200 \mathrm{WBCs}}
$$

Patient's WBC count $=8000 \mu \mathrm{L}$ (taken as average WBC count) (WHO, 2016). The mean values of detectable parasites will be determined and expressed as parasites per microliter of the original sample [23].

\subsubsection{Comparison of RDT Kits}

Two sets of doubling dilution of blood samples with parasitemia were made in serological tubes using physiological saline. Up to two doubling dilutions were prepared in each set to obtain a dilution range between 1:2 and 1:4. Two different kits of malarial were used for the titration of the two dilution sets in accordance with the manufacturer's instructions. Titer values were determined and expressed as the reciprocal of the highest dilution that shows positive result in the tubes.

A film was made by each of the respective dilutions. Parasite density was calculated at each of the dilution in other to detect the density at which each RDT kit can detect malaria. Known positive and negative control blood samples were treated with each of the kits.

\subsection{Statistical Analysis}

The data generated was analyzed and presented in form of tables and chart. Significant difference between the two methods employed during the study was checked using prism. Level of significance was set at $95 \%$ confidence limit (Pvalue of 0.05 level of significance).

\section{Results}

A total of 200 participants were enrolled in the study. Table 1 is the summary of the participants' demographic data. 113 $(56.5 \%)$ of them were males while $87(43.5 \%)$ were females. In this study, microscopy was used as the 'Gold Standard'. The study participants were categorized among the following age groups, 1-10 years (2), 11-20 years (28), 21-30 years (114) and 31-40 years (47), 41-50 years (4) and 51-60 years (5). A greater percentage $114(57 \%)$ belonged to the age group 21-30 years.

Table 1. Demographic Data Showing the Performance Of the three Diagnostic techniques.

\begin{tabular}{|c|c|c|c|c|c|c|}
\hline Characteristics & $\begin{array}{l}\text { Participants } \\
\text { Frequency (\%) }\end{array}$ & $\begin{array}{l}\text { Microscopy } \\
\text { Positives (\%) }\end{array}$ & $\begin{array}{l}\text { SD-Bioline } \\
\text { Positives (\%) }\end{array}$ & Negatives (\%) & $\begin{array}{l}\text { Care Start } \\
\text { Positives (\%) }\end{array}$ & Negatives (\%) \\
\hline \multicolumn{7}{|l|}{ Gender } \\
\hline Male & $113(56.5)$ & $113(56.5)$ & $86(43.0)$ & $27(13.5)$ & $71(35.5)$ & $42(21.0)$ \\
\hline Total & $200(100)$ & $200(100)$ & $159(79.5)$ & $41(20.5)$ & $139(69.5)$ & $61(30.5)$ \\
\hline \multicolumn{7}{|l|}{ Age Ranges (years) } \\
\hline $0-10$ & $2(1.0)$ & $2(1.0)$ & $2(1.0)$ & $0(0.0)$ & $1(0.5)$ & $1(0.5)$ \\
\hline $21-30$ & $114(57.0)$ & $114(57.0)$ & $88(44.0)$ & $26(13.0)$ & $78(39.0)$ & $36(18.0)$ \\
\hline $31-40$ & $47(23.5)$ & $47(23.5)$ & $42(21)$ & $5(2.5)$ & $13(6.5)$ & $34(17.0)$ \\
\hline $41-50$ & $4(2.0)$ & $4(2)$ & $4(0,0)$ & $0(0.0)$ & $4(2.0)$ & $0(0.0)$ \\
\hline $51-60$ & $5(2.5)$ & $5(2.5)$ & $4(2.0)$ & $1(0.5)$ & $2(1.0)$ & $3(1.5)$ \\
\hline Total & $200(100)$ & $200(100)$ & $159(79.5)$ & $41(20.5)$ & $113(56.5)$ & $87(43.5)$ \\
\hline
\end{tabular}

Table 2 Depicts the effectiveness of performance of the RDT kids compared to microscopy, it is worthy to note that only subjects tested positive by microscopy were employed in the study, hence all the $200(100 \%)$ subjects are positive for malaria through the microscopic examination while SDBioline and CareStart showed positive results of 159 (79.5\%) 
and $113(56.5 \%)$ respectively.

It also shows the comparison of the two RDTs with results of microscopy. There are $159(79.5 \%)$ of the subjects who were found to be malaria positive while 49 (20.5\%) of them were negative with SD-Bioline RDT. The number of subjects found to be malaria positive were $113(56.5 \%)$ and those negative were 87 (43.5\%) with care start RDT

Table 2. Effectiveness of Performance of RDT Kids Compared to Microscopy.

\begin{tabular}{llll}
\hline Diagnostic Tool & No. of Samples Tested (\%) & No. of positive Samples (\%) & No. of negative Samples (\%) \\
\hline Microscopy & $200(100)$ & $200(100)$ & $0(0.0)$ \\
SD-Bioline & $200(100)$ & $159(79.5)$ & $41(20.5)$ \\
Care start & $200(100)$ & $113(56.5)$ & $87(43.5)$ \\
\hline
\end{tabular}

Table 3 Show the effectiveness of performance of the RDT kids compared to Microscopy 200 participants were subjected to the study with microscopy having $100 \%$ of the samples tested positive, SD-Bioline $79.5 \%$ tested positive and $20.5 \%$ tested negative, Care Start $56.5 \%$ tested positive and $43.5 \%$ tested negative.

Table 3. Performance of RDT kids (for neat sample) compared to microscopy.

\begin{tabular}{lllll}
\hline Characteristics & No of positive (\%) & No of negative (\%) & Total (\%) & P value \\
\hline Microscopy & $200(100)$ & $0(0)$ & $200(100)$ & $<0.0001$ \\
SD-Bioline (neat sample) & $159(79.5)$ & $41(20.5)$ & $200(100)$ & \\
Care Start (neat sample) & $139(69.5)$ & $61(30.5)$ & $200(100)$ & \\
\hline
\end{tabular}

Table 4 Show the effectiveness of performance of the RDT kits for the 1 in 2 dilution (first dilution) compared to Microscopy, at this concentration of the sample $51.5 \%$ were tested and found them positive with SD-Bioline while $39.5 \%$ tested negative and $38.0 \%$ were tested positive while $62.0 \%$ tested negative with Care Start.

Table 4. Performance of RDT kids (for $1^{\text {st }}$ diluted sample) compared to microscopy.

\begin{tabular}{lllll}
\hline Characteristics & No of positive (\%) & No. of negative (\%) & Total (\%) & P value \\
\hline Microscopy & $200(100)$ & $0(0)$ & $200(100)$ & $<0.0001$ \\
SD-Bioline (1 $1^{\text {st }}$ dilution) & $103(51.5)$ & $97(39.5)$ & $200(100)$ & \\
CareStart (1 st $^{\text {dilution})}$ & $76(38.0)$ & $124(62.0)$ & $200(100)$ & \\
\hline
\end{tabular}

Table 5 Show the effectiveness of performance of the RDT kits for the 1 in 4 dilution compared to microscopy where all the $200(100 \%)$ participants in the study were tested positive. There were 159 (79.5\%) of the subjects who were found to be malaria positive while 49 (20.5\%) of them were negative with SD-Bioline RDT. The number of subjects found to be malaria positive were $113(56.5 \%)$ and those negative were 87 (43.5\%) with care start RDT.

Table 5. Performance of RDT kids (for $2^{\text {nd }}$ diluted sample) compared to microscopy.

\begin{tabular}{lllll}
\hline Characteristics & No of positive (\%) & No of negative (\%) & Total (\%) & P value \\
\hline Microscopy & $200(100)$ & $0(0)$ & $200(100)$ & $<0.0001$ \\
SD-Bioline (2 $2^{\text {nd }}$ dilution) & $41(20.5)$ & $159(79.5)$ & $200(100)$ & \\
CareStart $\left(2^{\text {nd }}\right.$ dilution) & $22(11.0)$ & $178(89)$ & $200(100)$ & \\
\hline
\end{tabular}

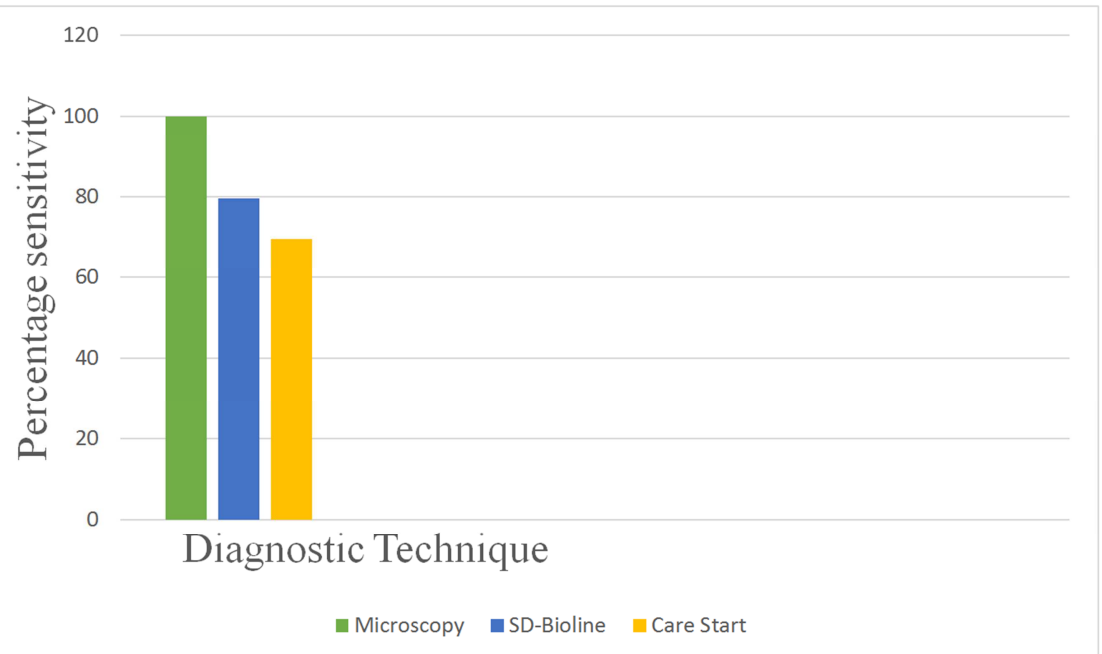

Figure 1. Depicts the correlation between the three diagnostic tools with the degree of performance on vertical axis and Diagnostic technique on the horizontal axis. 


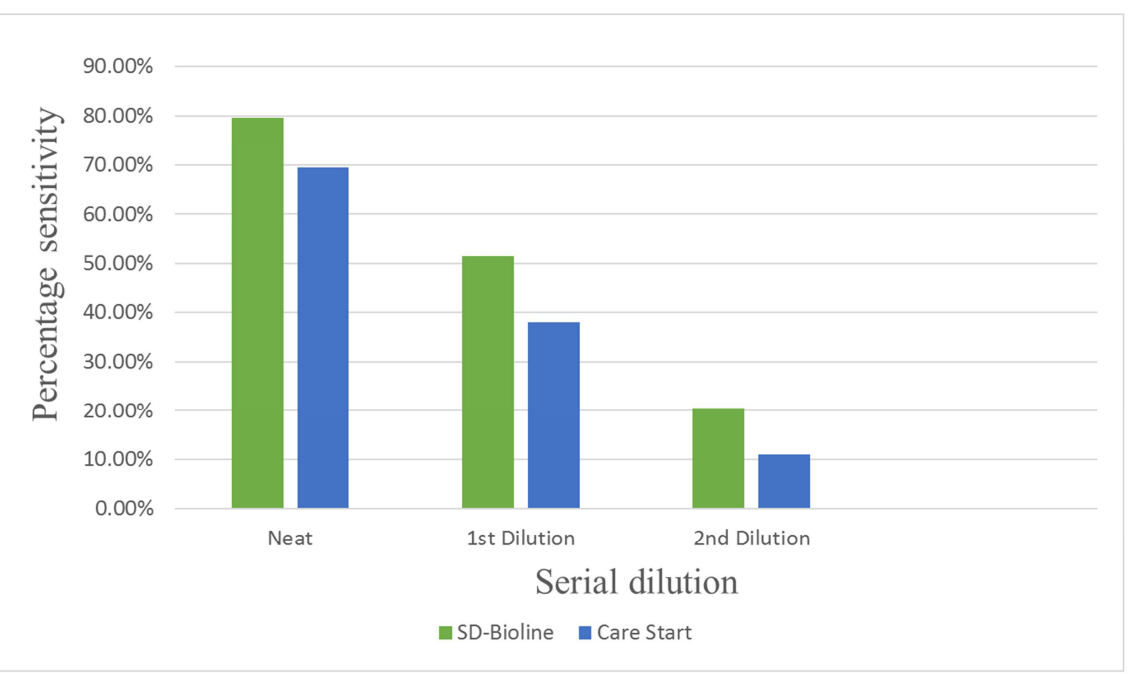

Figure 2. Depicts the correlation between the two RDTs at different concentration with the degree of performance on vertical axis and serial dilution on the horizontal axis.

\section{Discussion}

Malaria diagnosis is focused on detection of malaria parasite in the stained blood smears using microscopy or detection of parasite antigen using RDTs. One of the major disadvantages of RDTs is their inability to quantify parasite density [24].

This study has compared the performance of two RDTs using one histidine rich protein (SD-Bioline) and a combination of histidine rich protein and parasite lactose dehydrogenase enzyme (pLDH) RDT (CareStart).

As the results of the experiments illustrates, out of the 200 participants enrolled in the study, following gender as a variable, the male participants tend to have higher percentage with $113(56.5 \%), 86(43 \%)$ and $71(35.5 \%)$ tested positive by microscopy, SD-Bioline and Care Start respectively than females with $87(43.5 \%), 73(36.5 \%)$ and $68(34 \%)$ tested positive by microscopy. This could be due to the fact that males are more prone to mosquito bites in sitting areas. The age group with greater percentage is $21-30$ years with 114 $(56 \%), 88(44 \%)$ and $39(19.5 \%)$ tested positive by microscopy, SD-Bioline and CareStart respectively. This could be due to the fact that they are mostly expose to mosquito bites in reading places in the case of student and in business locations in case of business men. This is in consonant which another study [25].

The sensitivity of HRP-2 RDTs meets the WHO recommendation of $\geq 95 \%$ and is consistent with previous reports using Pf only RDTs in eastern Tanzania [26] and in Uganda (Murray et al., 2008). However, the results of HRP-2 kits are at variance with other studies which showed lower sensitivities. Studies conducted in Enugu Nigeria and in Ethiopia found a sensitivity of $69.7 \%$ for Global device RDT [27].

These have been ascribed to decreasing sensitivity with reduction in parasite density and thus patients with low density malaria parasites are missed, these could also be caused by failure of the parasite to express the antigen due to detection of the gene HRP-2 [28]. The detection of the two RDTs as negative with SD-Bioline (HRP-2) and CareStart (HRP-2/pLDH), whereas the corresponding microscopy was positive was encountered. This test has also been encountered in other studies as [29]. This could be as a result of low level of parasitaemia detetion of HRP-2 genes and the activity of the immune system of individual, which combats the antigens of the parasite in the peripheral blood, this makes the antigens to be absent, while present and seen in a stained film at microscopic examination.

Methodology of parasite density and diagnosing the blood samples at various dilutions enables the detection of the sensitivity of the kid to be measured [30]. SD-Bioline was more reactive at an average parasitaemia level (1:4 dilution) while Care Start was only weakly reactive at this same parasitaemia level. This shows that SD-Bioline has a more detection ability than CareStart. This result is in consonant with a study done previously in which SD-Bioline was reported to be reactive at an average parasitaemia level while care start was weakly reactive at the same parasitemia level [31].

The Study results revealed that the two RDTs showed an appreciable effectiveness though the SD-Bioline (HRP-2 RDT) showed a higher ability to detect malaria. The Underlying reason is that HRP-2 is a very stable antigen and has superior heat stability than pLDH which degrades easily [32]. The performance of the SD Bioline HRP-2 observed in the present study was agreed with previous studies with other HRP-2 based commercial RDTs such as Para check PfTM (Orchid Biomedical system) Binax Now MalariaTM (Binax, Inc, Inverness Medical Professional Diagnostic, USA).

\section{Conclusion}

There was a slight difference in sensitivity and specificity of SD-Bioline (HRP-2) and CareStart (HRP-2/pLDH) RDTs, also there was a significant mean difference paired for both and the microscopy standard and the RDTs which means 
their performances are not relatively the same. However, SDbioline can be used in the absence of microscopy because the study showed that it is more sensitive than care start in terms of detecting malaria antigen. The detection is slightly higher in SD-Bioline (HRP-2) RDT than in CareStart (HRP$2 / \mathrm{pLDH}$ ) but overall differences between the two RDTs was not very significant and performance was not comparable to microscopy, of which low parasite density is a great drawback to the diagnostic quality of both RDTs. This study Jhas found that both RDTs involved in the study to be a reliable diagnostic tool in malaria patients $\mathrm{N}$ with high parasitaemia values. Microscopy remains the gold standard in diagnosis and detection of parasites burden in malaria patients.

\section{Ethical Consideration}

Ethical approval to conduct the research was sought from the Research Ethics committee of Yobe State Specialist Hospital Damaturu, Yobe State.

\section{Availability of Data and Material}

All data generated or analysed during this study are included in this manuscript and are available from the corresponding author on reasonable request.

\section{Author Contributions}

M. I. conceived and designed the study. G. S. supervised the data collection. N. A. analysed. The data. M. K. wrote the first draft of the manuscript. All authors read and approved the final manuscript.

\section{Competing Interests}

The authors declare that they have no competing interest.

\section{Acknowledgements}

The authors wish to thank all patients who agree and to participate in this study. Many thanks also go to the staff in the parasitology laboratory of Specialist Hospital Yobe State, Adamawa State, Nigeria for their kind support and advice during this research work.

\section{References}

[1] Ito EE, Egwunyenga AO, Ake JE. Prevalence of malaria and human blood factors among patients in Ethiope East, Delta State, Nigeria. International Journal of Medicine and Biomedical Research. 2014; 3 (3): 191-201.

[2] Cha PI, Gurland B, Forrester JD. First Reported Case of Intussusception Caused by Escherichia coli O157: H7 in an Adult: Literature Review and Case Report. Surgical infections. 2019 Jan 1; 20 (1): 95-9.
[3] Karl S, Gurarie D, Zimmerman PA, King CH, Pierre TG, Davis TM. A sub-microscopic gametocyte reservoir can sustain malaria transmission. PloS one. 2011; 6 (6).

[4] Mtove G, Hendriksen IC, Amos B, Mrema H, Mandia V, Manjurano A, Sykes A, Hildenwall H, Whitty CJ, Reyburn H. Treatment guided by rapid diagnostic tests for malaria in Tanzanian children: safety and alternative bacterial diagnoses. Malaria journal. 2011 Dec 1; 10 (1): 290.

[5] Newell DG, Koopmans M, Verhoef L, Duizer E, Aidara-Kane A, Sprong H, Opsteegh M, Langelaar M, Threfall J, Scheutz F, van der Giessen J. Food-borne diseases - the challenges of 20 years ago still persist while new ones continue to emerge. International journal of food microbiology. 2010 May 30; 139: S3-15.

[6] Ullah Z, Noor B, Nadeem MF, Hayyat A, Khattak AA. Evaluation of immunochromatographic (ICT) assay and microscopy for malaria diagnosis in endemic district Dera Ismail Khan. Inter J Bio Sci. 2015; 6: 37-42.

[7] Matangila JR, Lufuluabo J, Ibalanky AL, da Luz RA, Lutumba P, Van Geertruyden JP. Asymptomatic Plasmodium falciparum infection is associated with anaemia in pregnancy and can be more cost-effectively detected by rapid diagnostic test than by microscopy in Kinshasa, Democratic Republic of the Congo. Malaria journal. 2014 Dec; 13 (1): 132.

[8] Moody A. Rapid diagnostic tests for malaria parasites. Clinical microbiology reviews. 2002 Jan 1; 15 (1): 66-78.

[9] Rahimpour S, Nasiri-Toosi M, Khalili H, Daryani NE, Taromlou N, Kazem M, Azizi Z. A Triple Blinded, Randomized, Placebo-Controlled Clinical Trial to Evaluate the Efficacy and Safety of Oral Vancomycin in Primary Sclerosing Cholangitis: a Pilot Study. Journal of Gastrointestinal \& Liver Diseases. 2016 Dec 1; 25 (4).

[10] Juneau C, Bates GW. Reproductive outcomes after medical and surgical management of ectopic pregnancy. Clinical obstetrics and gynecology. 2012 Jun 1; 55 (2): 455-60.

[11] Skandarajah A, Reber CD, Switz NA, Fletcher DA. Quantitative imaging with a mobile phone microscope. PloS one. 2014; 9 (5).

[12] Uzochukwu BS, Chiegboka LO, Enwereuzo C, Nwosu U, Okorafor D, Onwujekwe OE, Uguru NP, Sibeudu FT, Ezeoke OP. Examining appropriate diagnosis and treatment of malaria: availability and use of rapid diagnostic tests and artemisininbased combination therapy in public and private health facilities in south east Nigeria. BMC Public Health. 2010 Dec 1; 10 (1): 486.

[13] World Health Organization. Guidelines for the treatment of malaria. World Health Organization; 2015 Aug 13.

[14] Talman AM, Duval L, Legrand E, Hubert V, Yen S, Bell D, Le Bras J, Ariey F, Houze S. Evaluation of the intra-and interspecific genetic variability of Plasmodium lactate dehydrogenase. Malaria journal. 2007 Dec; 6 (1): 140.

[15] Italiano CM, Wong KT, AbuBakar S, Lau YL, Ramli N, Omar SF, Bador MK, Tan CT. Sarcocystis nesbitti causes acute, relapsing febrile myositis with a high attack rate: description of a large outbreak of muscular sarcocystosis in Pangkor Island, Malaysia, 2012. PLoS neglected tropical diseases. 2014 May; 8 (5). 
[16] Ferreira MU, Castro MC. Challenges for malaria elimination in Brazil. Malaria journal. 2016 Dec; 15 (1): 284.

[17] Pembele GN, Rivero LR, Fraga J. Detection and species identification of malaria parasites by nested-PCR: comparison with light microscopy and with SD BIOLINE Malaria Ag test in Luanda, Angola. Int J Trop Dis Health. 2015; 10 (1): 1-3.

[18] Obono O, Omoluabi E. Technical and political aspects of the 2006 Nigerian population and housing Census. African Population Studies. 2014 Mar 24; 27 (2): 249-62.

[19] Ahmed TA. Isolation of Pseudomonas species can be Transmitted by Musca domestica Collected from Restaurants in Wad Medani, Sudan (2016) (Doctoral dissertation, University of Gezira).

[20] Ugwu CC. Evaluation of Bacteriological Quality of Dried Cray Fish (Astacus leptodactylus) Sold at Ogbete Main Market in Enugu Metropolis, Nigeria. Int. J. Curr. Microbiol. App. Sci. 2019; 8 (9): 2643-8.

[21] Yahaya H, Taura D, Gwarzo M, Ibrahim A, Ali B, Muhammad A. Diversity of respiratory yeasts from suspected pulmonary tuberculosis patients. Scholars Journal of Applied Medical Sciences. 2014; 2: 3145-50.

[22] Karamba KI, Kawo AH, Dabo NT, Mukhtar MD. A survey of avian malaria parasite in Kano State, Northern Nigeria. Int $\mathbf{J}$ Biotechnol Mol Biol Res. 2012; 3 (1): 8-14.

[23] Abubakar A, Emokpae A, Sarkinfada F. Evaluation of the Detection Threshold of Three Immonochromotographic Rapid Diagnostic Test (RDT) Kits for Falciparum...

[24] TAKALMAWA H. AG ABUBAKARII, MA EMOKPAE2 F. SARKINFADA3 AH KWARU1, T. OFOLU1, O. IMORUI• NS MAGAJP, AH ARZAP, D. MUSA LAF SCHOTT6. Journal of Medical Laboratory Science. 2004; 3 (2).

[25] Aliyu AA, Dahiru T, Ladan AM, Shehu AU, Abubakar AA,
Oyefabi AM, Yahaya SS. Knowledge, sources of information, and risk factors for sexually transmitted infections among secondary school youth in Zaria, Northern Nigeria. Journal of Medicine in the Tropics. 2013 Dec 23; 15 (2): 102.

[26] Mtove G, Amos B, Nadjm B, Hendriksen IC, Dondorp AM, Mwambuli A, Kim DR, Ochiai RL, Clemens JD, Von Seidlein L, Reyburn H. Decreasing incidence of severe malaria and community-acquired bacteraemia among hospitalized children in Muheza, north-eastern Tanzania, 2006-2010. Malaria journal. $2011 \mathrm{Dec} ; 10$ (1): 320.

[27] Dozie UW, Chukwuocha UM. Journal of Tropical Diseases. J Trop Dis. 2016; 4 (2): 1000201

[28] Wurtz N, Fall B, Bui K, Pascual A, Fall M, Camara C, Diatta B, Fall KB, Mbaye PS, Diémé Y, Bercion R. Pfhrp2 and pfhrp3 polymorphisms in Plasmodium falciparum isolates from Dakar, Senegal: impact on rapid malaria diagnostic tests. Malaria journal. 2013 Dec 1; 12 (1): 34.

[29] Weiss NS. Clinical epidemiology: the study of the outcome of illness. Monographs in Epidemiology and; 2006.

[30] Erkens CG, Kamphorst M, Abubakar I, Bothamley GH, Chemtob D, Haas W, Migliori GB, Rieder HL, Zellweger JP, Lange C. Tuberculosis contact investigation in low prevalence countries: a European consensus.

[31] Adamou A, Arbeille P, Coulibaly K, Diouf MB, Dzodzomenyo M, Fall K, Fossbakk BM, Guèye PM, Kana MA, Karam F, Kargirwar V. Dakar, Senegal \& Kololi and Tendaba, The Gambia Saturday 21 st to Friday 27 th April, 2007.

[32] Madanitsa M. The Potential of Intermittent Screening and Treatment with Dihydroartemisinin-Piperaquine for the control of malaria in pregnancy in areas with high sulphadoxine-pyrimethamine resistance (Doctoral dissertation, University of Liverpool). 